\title{
SEMIGROUPS OF CENTERED UPFAMILIES ON FINITE MONOGENIC SEMIGROUPS
}

\section{VOLODYMYR GAVRYLKIV}

Vasyl Stefanyk Precarpathian National University

Ivano-Frankivsk

Ukraine

e-mail: vgavrylkiv@yahoo.com

\begin{abstract}
Given a finite monogenic semigroup $S$, we study the minimal ideal, the center, left cancelable, and right cancelable elements of the extension $N_{<\omega}(S)$

consisting of centered upfamilies on $S$ and characterize monogenic semigroups whose extensions are commutative.
\end{abstract}

\section{Introduction}

This paper is devoted to describing the structure of extensions $N_{<\omega}(S)$ of monogenic semigroups $S$. The thorough study of various extensions of semigroups was started in [11] and continued in [1]-[8], [12]-[15]. The largest among these extensions is the semigroup $v(S)$ of all upfamilies on $S$. A family $\mathcal{M}$ of nonempty subsets of a set $X$ is called an upfamily if for each set $A \in \mathcal{M}$ any subset $B \supset A$ of $X$ belongs to $\mathcal{M}$. Each family $\mathcal{B}$ of nonempty subsets of $X$ generates the upfamily

2010 Mathematics Subject Classification: 20M10, 20M14, 20M17, 20M18, 54B20.

Keywords and phrases: monogenic semigroup, centered upfamily, zero, minimal ideal, commutative.

Received September 19, 2016; Revised November 12, 2016

(C) 2016 Scientific Advances Publishers 
$\langle B \subset X: B \in \mathcal{B}\rangle=\{A \subset X: \exists B \in \mathcal{B}(B \subset A)\}$. An upfamily $\mathcal{F}$ that is closed under taking finite intersections is called a filter. A filter $\mathcal{U}$ is called an ultrafilter if $\mathcal{U}=\mathcal{F}$ for any filter $\mathcal{F}$ containing $\mathcal{U}$. The family $\beta(X)$ of all ultrafilters on a set $X$ is called the Stone-Čech compactification of $X$ (see [16], [19]). An ultrafilter $\langle\{x\}\rangle$, generated by a singleton $\{x\}$, $x \in X$, is called principal. Identifying each point $x \in X$ with the principal ultrafilter $\langle\{x\}\rangle$ we obtain the inclusions $X \subset \beta(X) \subset v(X)$. It was shown in [11] that any associative binary operation $*: S \times S \rightarrow S$ can be extended to an associative binary operation $\circ: v(S) \times v(S) \rightarrow v(S)$ by the formula

$$
\mathcal{L} \circ \mathcal{M}=\left\langle\bigcup_{a \in L} a * M_{a}: L \in \mathcal{L},\left\{M_{a}\right\}_{a \in L} \subset \mathcal{M}\right\rangle,
$$

for upfamilies $\mathcal{L}, \mathcal{M} \in v(S)$. In this case, the Stone-Čech compactification $\beta(S)$ is a subsemigroup of the semigroup $v(S)$. The semigroup $v(S)$ contains many other important extensions of $S$. In particular, it contains the semigroup $N_{<\omega}(S)$ of centered upfamilies. An upfamily $\mathcal{L} \in v(S)$ is called centered if $\bigcap \mathcal{F} \neq \emptyset$ for any finite subfamily $\mathcal{F} \subset \mathcal{L}$.

Each map $f: X \rightarrow Y$ induces the map

$$
N_{<\omega} f: N_{<\omega}(X) \rightarrow N_{<\omega}(Y), \quad N_{<\omega} f: \mathcal{M} \mapsto\langle f(M) \subset Y: M \in \mathcal{M}\rangle,
$$

see $[10]$.

A nonempty subset $I$ of a semigroup $(S, *)$ is called an ideal (resp., a right ideal, a left ideal) if $I * S \cup S * I \subset I$ (resp., $I * S \subset I, S * I \subset I$ ). An element $z$ of a semigroup $(S, *)$ is called a zero (resp., a left zero, a right zero) in $S$ if $a * z=z * a=z$ (resp., $z * a=z, a * z=z$ ) for any $a \in S$. It is clear that $z \in S$ is a zero (resp., a left zero, a right zero) in $S$ if and only if the singleton $\{z\}$ is an ideal (resp., a right ideal, a left ideal) in $S$. An ideal $I \subset S$ is called minimal if any ideal of $S$ that lies in $I$ coincides with $I$. By analogy, we define minimal left and minimal right 
ideals of $S$. The union $K(S)$ of all minimal left (right) ideals of $S$ coincides with the minimal ideal of $S$, see [16, Theorem 2.8]. A semigroup $(S, *)$ is said to be a right zero semigroup if $a * b=b$ for any $a, b \in S$. A map $\varphi: S \rightarrow T$ between semigroups $(S, *)$ and $(T, \circ)$ is called a homomorphism if $\varphi(a * b)=\varphi(a) \circ \varphi(b) \quad$ for any $\quad a, b \in S$. A homomorphism $\varphi: S \rightarrow I$ from a semigroup $S$ onto an ideal $I \subset S$ is called a retraction if $\varphi(a)=a$ for any element $a \in I$. An element $a$ of a semigroup $S$ is called left cancelable (resp., right cancelable) if for any elements $x, y \in S$ the equality $a x=a y$ (resp., $x a=y a$ ) implies $x=y$. This is equivalent to saying that the left (resp., right) shift $l_{a}: S \rightarrow S$, $l_{a}: x \mapsto a * x$ (resp., $r_{a}: S \rightarrow S, r_{a}: x \mapsto x * a$ ) is injective. A semigroup $S$ is called left (right) cancellative if all elements of $S$ are left (right) cancelable. A semigroup that is both left and right cancellative is said to be cancellative. By definition, the center of a semigroup $S$ is the set $C(S)=\{a \in S: \forall s \in S(s a=a s)\}$.

A semigroup $\langle a\rangle=\left\{a^{n}\right\}_{n \in \mathbb{N}}$ generated by a single element a is called monogenic or cyclic. If a monogenic semigroup is infinite, then it is isomorphic to the additive semigroup $\mathbb{N}$. A finite monogenic semigroup $S=\langle a\rangle$ also has very simple structure (see [9]). There are positive integer numbers $r$ and $m$ called the index and the period of $S$ such that

- $S=\left\{a, a^{2}, \ldots, a^{m+r-1}\right\}$ and $m+r-1=|S|$

- for any $i, j \in \omega$ the equality $a^{r+i}=a^{r+j}$ holds if and only if $i \equiv j$ $\bmod m$;

- $C_{m}=\left\{a^{r}, a^{r+1}, \ldots, a^{m+r-1}\right\}$ is the minimal ideal, a cyclic and maximal subgroup of $S$ with the neutral element $e=a^{n} \in C_{m}$, where $m$ divides $n$.

From now on we denote by $C_{r, m}$ a finite monogenic semigroup of index $r$ and period $m$, and maximal subgroup of $C_{r, m}$ is denoted by $C_{m}$. 


\section{Homomorphisms, Zeros and Minimal Ideals}

Proposition 2.1. For any homomorphism $\varphi: S \rightarrow T$ between semigroups $\left(S, *_{1}\right)$ and $\left(T, *_{2}\right)$ the induced map $N_{<\omega} \varphi: N_{<\omega}(S) \rightarrow$ $N_{<\omega}(T)$ is a homomorphism of the semigroups $\left(N_{<\omega}(S), \circ_{1}\right)$ and $\left(N_{<\omega}(T), \circ_{2}\right)$.

Proof. Given two centered upfamilies $\mathcal{L}, \mathcal{M} \in N_{<\omega}(S)$ observe that

$$
\begin{aligned}
N_{<\omega} \varphi\left(\mathcal{L}{ }^{\circ}\right. & \mathcal{M})=N_{<\omega} \varphi\left(\left\langle\bigcup_{x \in L} x *_{1} M_{x}: L \in \mathcal{L},\left\{M_{x}\right\}_{x \in L} \subset \mathcal{M}\right\rangle\right) \\
= & \left\langle\varphi\left(\bigcup_{x \in L} x *_{1} M_{x}\right): L \in \mathcal{L},\left\{M_{x}\right\}_{x \in L} \subset \mathcal{M}\right\rangle \\
= & \left\langle\bigcup_{x \in L} \varphi(x) *_{2} \varphi\left(M_{x}\right): L \in \mathcal{L},\left\{M_{x}\right\}_{x \in L} \subset \mathcal{M}\right\rangle \\
= & \left\langle\bigcup_{x \in \varphi(L)} x *_{2} \varphi\left(M_{x}\right): L \in \mathcal{L},\left\{\varphi\left(M_{x}\right)\right\}_{x \in \varphi(L)} \subset N_{<\omega} \varphi(\mathcal{M})\right\rangle \\
= & \langle\varphi(L): L \in \mathcal{L}\rangle \circ_{2}\langle\varphi(M): M \in \mathcal{M}\rangle=N_{<\omega} \varphi(\mathcal{L}) \circ_{2} N_{<\omega} \varphi(\mathcal{M}) .
\end{aligned}
$$

Let us note that for a subsemigroup $T$ of a semigroup $S$ the homomorphism $i: N_{<\omega}(T) \rightarrow N_{<\omega}(S), i: \mathcal{A} \rightarrow\langle\mathcal{A}\rangle_{S}$ is injective, and thus we can identify the semigroup $N_{<\omega}(T)$ with the subsemigroup $i\left(N_{<\omega}(T)\right) \subset N_{<\omega}(S)$. Therefore, for each family $\mathcal{B}$ of nonempty subsets of $T$, we identify the upfamilies

$$
\langle\mathcal{B}\rangle_{T}=\{A \in T \mid \exists B \in \mathcal{B}(B \subset A)\} \in N_{<\omega}(T),
$$

and

$$
\langle\mathcal{B}\rangle_{S}=\{A \in S \mid \exists B \in \mathcal{B}(B \subset A)\} \in N_{<\omega}(S) .
$$


Lemma 2.2. Let I be an ideal of a semigroup $S$. If a map $\varphi: S \rightarrow I$ is a retraction, then the map $N_{<\omega} \varphi: N_{<\omega}(S) \rightarrow N_{<\omega}(I)$ is a retraction too.

Proof. Indeed, let $\mathcal{A} \in N_{<\omega}(I), \mathcal{M} \in N_{<\omega}(S)$, then $\mathcal{A} \circ \mathcal{M}=$ $\left\langle\bigcup_{a \in A} a * M_{a}: A \in \mathcal{A}, A \subset I,\left\{M_{a}\right\}_{a \in A} \subset \mathcal{M}\right\rangle=\left\langle\bigcup_{a \in A} a * M_{a}: A \in \mathcal{A}\right.$, $\left.\left\{M_{a}\right\}_{a \in A} \subset \mathcal{M}, \bigcup_{a \in A} a * M_{a} \subset I\right\rangle \in N_{<\omega}(I)$. By analogy $\mathcal{M} \circ \mathcal{A} \in N_{<\omega}(I)$, and therefore $N_{<\omega}(I)$ is an ideal of the semigroup $N_{<\omega}(S)$. If $\mathcal{A} \in N_{<\omega}(I)$, then $N_{<\omega} \varphi(\mathcal{A})=\langle\varphi(A): A \subset I, A \in \mathcal{A}\rangle=\langle A: A \subset I, A \in \mathcal{A}\rangle$ $=\{A \in \mathcal{A}: A \subset I\}=\mathcal{A}$ and hence $N_{<\omega} \varphi$ is a retraction.

Let $e$ be the neutral element of the maximal subgroup $C_{m}$ of a monogenic semigroup $C_{r, m}$.

Lemma 2.3. The map $\varphi: C_{r, m} \rightarrow C_{m}, \varphi(x)=e x$ is a retraction and $\varphi(x) y=x y$ for any $x \in C_{r, m}$ and $y \in C_{m}$.

Proof. Since the semigroup $C_{m}$ is an ideal of the semigroup $C_{r, m}$, $\varphi(x)=e x \in C_{m}$. Consequently, $\varphi(x y)=e x y=e e x y=e x e y=\varphi(x) \varphi(y)$ for any $x, y \in C_{r, m}$ and $\varphi(x)=e x=x$ for $x \in C_{m}$. Hence the map $\varphi: C_{r, m} \rightarrow C_{m}$ is a retraction. Further for any $x \in C_{r, m}$ and $y \in C_{m}$, we have that $x y \in C_{m}$, and therefore $\varphi(x y)=x y$. On the other hand, $\varphi(x y)=\varphi(x) \varphi(y)=\varphi(x) y$, since $y \in C_{m}$.

Proposition 2.4. For each finite monogenic semigroup $C_{r, m}$, the centered upfamily $\left\langle C_{m}\right\rangle$ is the zero of the semigroup $N_{<\omega}\left(C_{r, m}\right)$.

Proof. Let $\varphi: C_{r, m} \rightarrow C_{m}$ be the retraction from Lemma 2.3. Since $x F \supset x C_{m}=\varphi(x) C_{m}=C_{m}$ for each $x \in C_{r, m}$ and $F \in\left\langle C_{m}\right\rangle$, then $x F \in\left\langle C_{m}\right\rangle$ and $\left\langle C_{m}\right\rangle$ is a right zero according to Proposition 1 from [15]. 
We shall show that $\left\langle C_{m}\right\rangle$ is a left zero, that is $\left\langle C_{m}\right\rangle \circ \mathcal{A}=\left\langle C_{m}\right\rangle$ for each $\mathcal{A} \in N_{<\omega}\left(C_{r, m}\right)$. Since $C_{m} A=C_{m} \varphi(A)=C_{m}$ for $A \in \mathcal{A}$, then $\left\langle C_{m}\right\rangle \subset\left\langle C_{m}\right\rangle \circ \mathcal{A}$. If $M \in\left\langle C_{m}\right\rangle \circ \mathcal{A}$, then $M \supset \bigcup_{g \in C_{m}} g A_{g},\left\{A_{g}\right\}_{g \in C_{m}} \subset \mathcal{A}$. Since $\mathcal{A}$ is a centered upfamily and $C_{m}$ is finite, then there exists $a \in \bigcap_{g \in C_{m}} A_{g} . \quad$ Therefore, $\quad M \supset \bigcup_{g \in C_{m}} g\{a\}=C_{m} a=C_{m} \varphi(a)=C_{m}$ and $M \in\left\langle C_{m}\right\rangle$.

Since an element $z$ of a semigroup $S$ is a zero in $S$ if and only if the singleton $\{z\}$ is an ideal in $S$, Proposition 2.4 implies the following:

Proposition 2.5. The minimal ideal of the semigroup $N_{<\omega}\left(C_{r, m}\right)$ is singleton, that is $K\left(N_{<\omega}\left(C_{r, m}\right)\right)=\left\{\left\langle C_{m}\right\rangle\right\}$.

\section{Commutativity and the Center}

Theorem 3.1. A finite monogenic semigroup $C_{r, m}=\left\{a, \ldots, a^{r}, \ldots\right.$, $\left.a^{m+r-1} \mid a^{r+m}=a^{r}\right\}$ of order $m+r-1$ has commutative extension $N_{<\omega}\left(C_{r, m}\right)$ if and only if

$$
(r, m) \in\{(1,1),(1,2),(2,1),(2,2),(3,1)\} .
$$

Proof. It was proved in the paper [15] that the extension $N_{<\omega}(G)$ of a group $G$ is commutative if and only if $|G| \leq 2$. Since for $m>2$, the extension $N_{<\omega}\left(C_{r, m}\right)$ contains a noncommutative subsemigroup $N_{<\omega}\left(C_{m}\right)$, then $N_{<\omega}\left(C_{r, m}\right)$ is not commutative. So it is sufficient to consider only finite monogenic semigroups of period $m \leq 2$.

If index $r=1$, then $C_{r, m}$ is a cyclic group of order $m$, and thus for $r=1$, the semigroup $N_{<\omega}\left(C_{r, m}\right)$ is commutative if and only if $m \leq 2$. 
If $r=2, m \in\{1,2\}$, then the product $x y$ of any two elements of $C_{r, m}$ is contained in the maximal subgroup $x y \in C_{m}$, and thus $x y=\varphi(x y)=$ $\varphi(x) \varphi(y)$, where $\varphi: C_{r, m} \rightarrow C_{m}$ is the retraction $\varphi: s \rightarrow e s$. Since extensions $N_{<\omega}(G)$ of groups $G$ of order 1 and 2 are commutative and the map $N_{<\omega} \varphi: N_{<\omega}\left(C_{r, m}\right) \rightarrow N_{<\omega}\left(C_{m}\right)$ is a retraction according to Proposition 2.2, then

$$
\mathcal{A} \circ \mathcal{B}=N_{<\omega} \varphi(\mathcal{A}) \circ N_{<\omega} \varphi(\mathcal{B})=N_{<\omega} \varphi(\mathcal{B}) \circ N_{<\omega} \varphi(\mathcal{A})=\mathcal{B} \circ \mathcal{A},
$$

for any $\mathcal{A}, \mathcal{B} \in N_{<\omega}\left(C_{r, m}\right)$. Consequently, the semigroups $N_{<\omega}\left(C_{2,1}\right)$ and $N_{<\omega}\left(C_{2,2}\right)$ are commutative.

Consider the semigroup $C_{3,1}=\left\{a, a^{2}, a^{3}: a^{4}=a^{3}\right\}$. The semigroup $N_{<\omega}\left(C_{3,1}\right)$ contains 10 elements.

Let us introduce the notation

$$
\left.\right|_{y} ^{x}=\left.\right|_{x} ^{y}=\langle\{x, y\}\rangle, \quad \vee_{x}=\left\{F \subset C_{3,1}:|F| \geq 2, x \in F\right\} .
$$

Also the principal ultrafilter $\langle\{e\}\rangle$ and the upfamily $\left\{C_{3,1}\right\}$ are identified with $e$ and $C_{3,1}$, respectively.

The following Cayley table implies the commutativity of $N_{<\omega}\left(C_{3,1}\right)$. 


\begin{tabular}{|c|c|c|c|c|c|c|c|c|c|c|}
\hline 。 & $a$ & $a^{2}$ & $a^{3}$ & $\left.\right|_{a} ^{a^{2}}$ & $\left.\right|_{a} ^{a^{3}}$ & $\left.\right|_{a^{2}} ^{a^{3}}$ & $\vee_{a}$ & $\vee_{a^{2}}$ & $\vee_{a^{3}}$ & $C_{3,1}$ \\
\hline$a$ & $a^{2}$ & $a^{3}$ & $a^{3}$ & $\left.\right|_{a^{2}} ^{a^{3}}$ & $\mid \begin{array}{l}a^{3} \\
a^{2}\end{array}$ & $a^{3}$ & $\left.\right|_{a^{2}} ^{a^{3}}$ & $a^{3}$ & $a^{3}$ & $\left.\right|_{a^{2}} ^{a^{3}}$ \\
\hline$a^{2}$ & $a^{3}$ & $a^{3}$ & $a^{3}$ & $a^{3}$ & $a^{3}$ & $a^{3}$ & $a^{3}$ & $a^{3}$ & $a^{3}$ & $a^{3}$ \\
\hline$a^{3}$ & $a^{3}$ & $a^{3}$ & $a^{3}$ & $a^{3}$ & $a^{3}$ & $a^{3}$ & $a^{3}$ & $a^{3}$ & $a^{3}$ & $a^{3}$ \\
\hline$\left.\right|_{a} ^{a^{2}}$ & $\left.\right|_{a^{2}} ^{a^{3}}$ & $a^{3}$ & $a^{3}$ & $\mid \begin{array}{l}a^{3} \\
a^{2}\end{array}$ & $\mid \begin{array}{l}a^{3} \\
a^{2}\end{array}$ & $a^{3}$ & $\left.\right|_{a^{2}} ^{a^{3}}$ & $a^{3}$ & $a^{3}$ & $\left.\right|_{a^{2}} ^{a^{3}}$ \\
\hline$\left.\right|_{a} ^{a^{3}}$ & $\left.\right|_{a^{2}} ^{a^{3}}$ & $a^{3}$ & $a^{3}$ & $\mid \begin{array}{l}a^{3} \\
a^{2}\end{array}$ & $\mid \begin{array}{l}a^{3} \\
a^{2}\end{array}$ & $a^{3}$ & $\left.\right|_{a^{2}} ^{a^{3}}$ & $a^{3}$ & $a^{3}$ & $\left.\right|_{a^{2}} ^{a^{3}}$ \\
\hline$\left.\right|_{a^{2}} ^{a^{3}}$ & $a^{3}$ & $a^{3}$ & $a^{3}$ & $a^{3}$ & $a^{3}$ & $a^{3}$ & $a^{3}$ & $a^{3}$ & $a^{3}$ & $a^{3}$ \\
\hline$\vee_{a}$ & $\left.\right|_{a^{2}} ^{a^{3}}$ & $a^{3}$ & $a^{3}$ & $\left.\right|_{a^{2}} ^{a^{3}}$ & $\mid \begin{array}{l}a^{3} \\
a^{2}\end{array}$ & $a^{3}$ & $\left.\right|_{a^{2}} ^{a^{3}}$ & $a^{3}$ & $a^{3}$ & $\left.\right|_{a^{2}} ^{a^{3}}$ \\
\hline$\vee_{a^{2}}$ & $a^{3}$ & $a^{3}$ & $a^{3}$ & $a^{3}$ & $a^{3}$ & $a^{3}$ & $a^{3}$ & $a^{3}$ & $a^{3}$ & $a^{3}$ \\
\hline$\vee_{a^{3}}$ & $a^{3}$ & $a^{3}$ & $a^{3}$ & $a^{3}$ & $a^{3}$ & $a^{3}$ & $a^{3}$ & $a^{3}$ & $a^{3}$ & $a^{3}$ \\
\hline$C_{3,1}$ & $\left.\right|_{a^{2}} ^{a^{3}}$ & $a^{3}$ & $a^{3}$ & $\left.\right|_{a^{3}} ^{a^{3}}$ & $\mid \begin{array}{l}a^{3} \\
a^{2}\end{array}$ & $a^{3}$ & $\left.\right|_{a^{2}} ^{a^{3}}$ & $a^{3}$ & $a^{3}$ & $\left.\right|_{a^{2}} ^{a^{3}}$ \\
\hline
\end{tabular}

Consider the semigroup $C_{3,2}=\left\{a, a^{2}, a^{3}, a^{4}: a^{5}=a^{3}\right\}$ and centered upfamilies $\mathcal{A}=\left\langle\left\{a, a^{2}\right\}\right\rangle, \mathcal{B}=\left\langle\left\{a, a^{2}\right\},\left\{a, a^{3}\right\}\right\rangle$ of the semigroup $N_{<\omega}\left(C_{3,2}\right)$. Since

$$
\mathcal{A} \circ \mathcal{B}=\left\langle\left\{a^{2}, a^{3}\right\}\right\rangle \neq\left\langle\left\{a^{2}, a^{3}, a^{4}\right\}\right\rangle=\mathcal{B} \circ \mathcal{A},
$$

then the semigroup $N_{<\omega}\left(C_{3,2}\right)$ is not commutative.

Let $r \geq 4$. Consider centered upfamilies $\mathcal{A}=\left\langle\left\{a, a^{2}\right\}\right\rangle$ and $\mathcal{B}=\langle\{a$, $\left.\left.a^{2}\right\},\left\{a^{2}, a^{3}\right\}\right\rangle$. We have that $a^{3} \notin a\left\{a, a^{3}\right\} \cup a^{2}\left\{a^{2}, a^{3}\right\} \in \mathcal{A} \circ \mathcal{B}$, but $a^{3} \in F$ for any $F \in \mathcal{B} \circ \mathcal{A}$. Consequently, $\mathcal{A} \circ \mathcal{B} \neq \mathcal{B} \circ \mathcal{A}$ and for each $r \geq 4$ a semigroup $N_{<\omega}\left(C_{r, m}\right)$ is not commutative. 
Let us study the center of the semigroup $N_{<\omega}\left(C_{r, m}\right)$. Since monogenic semigroups are commutative, then the formula

$$
\mathcal{L} \circ \mathcal{M}=\left\langle\bigcup_{a \in L} a * M_{a}: L \in \mathcal{L},\left\{M_{a}\right\}_{a \in L} \subset \mathcal{M}\right\rangle
$$

implies that the center $C\left(N_{<\omega}\left(C_{r, m}\right)\right)$ of a semigroup $N_{<\omega}\left(C_{r, m}\right)$ contains all principal ultrafilters. It was shown in [15] that $C\left(N_{<\omega}(G)\right)$ $=\{\langle\{g\}\rangle: g \in G\} \cup\{\langle G\rangle\}$ for a finite Abelian group $G$, that is the center $C\left(N_{<\omega}(G)\right)$ of the semigroup $N_{<\omega}(G)$ is isomorphic to $G^{0}$. Therefore, $C\left(N_{<\omega}\left(C_{m}\right)\right) \cong\left(C_{m}\right)^{0}$.

Lemma 3.2. Let $\varphi: S \rightarrow I$ be a retraction of a semigroup $S$ on an ideal I. If $a \in C(S)$, then $\varphi(a) \in C(I)$.

Proof. Indeed, for any $x \in I$, we have

$$
\varphi(a) x=\varphi(a) \varphi(x)=\varphi(a x)=\varphi(x a)=\varphi(x) \varphi(a)=x \varphi(a) .
$$

Theorem 3.3. For each finite monogenic semigroup $C_{r, m}$, the center of the semigroup $N_{<\omega}\left(C_{r, m}\right)$ contains centered upfamilies that are neither principal ultrafilters nor the zero of $N_{<\omega}\left(C_{r, m}\right)$. The center of the semigroup $N_{<\omega}\left(C_{2, m}\right)$ contains $m+5$ elements.

Proof. Since by Lemmas 2.3 and 2.2 the maps $\varphi: C_{r, m} \rightarrow C_{m}$, $\varphi(x)=e x \quad$ and $\quad N_{<\omega} \varphi: N_{<\omega}\left(C_{r, m}\right) \rightarrow N_{<\omega}\left(C_{m}\right)$ are retractions and $\left\langle C_{m}\right\rangle$ is the zero of $N_{<\omega}\left(C_{r, m}\right)$ and $N_{<\omega}\left(C_{m}\right)$, then Lemma 3.2 implies that

$$
C\left(N_{<\omega}\left(C_{r, m}\right)\right) \subset\left(N_{<\omega} \varphi\right)^{-1}\left(\left\{\langle\{g\}\rangle: g \in C_{m}\right\} \cup\left\{\left\langle C_{m}\right\rangle\right\}\right) .
$$


Let $a$ be a generator of a semigroup $C_{r, m}$. Consider elements $a^{r-1}$ and $\varphi\left(a^{r-1}\right)=e a^{r-1} \neq a^{r-1}$. We claim that centered upfamilies $\mathcal{A}=\left\langle\left\{a^{r-1}\right.\right.$, $\left.\left.e a^{r-1}\right\}\right\rangle, \mathcal{B}=\left\langle\left\{a^{r-1}\right\} \cup C_{m}\right\rangle$, and $\mathcal{C}=\left\langle\left\{a^{r-1}\right\} \cup C_{m} \backslash\left\{e a^{r-1}\right\}\right\rangle$ are central elements of the semigroup $N_{<\omega}\left(C_{r, m}\right)$.

Indeed, since $a^{r-1} x \in C_{m}=\left\{a^{r}, \ldots, a^{r+m-1}\right\}$ for each $x \in C_{r, m}$, then $a^{r-1} x=\varphi\left(a^{r-1} x\right)=\varphi\left(a^{r-1}\right) \varphi(x)$. On the other hand, since $C_{m}$ is an ideal of $C_{r, m}$, then $\varphi\left(a^{r-1}\right) x \in C_{m}$ and

$$
\varphi\left(a^{r-1}\right) x=\varphi\left(\varphi\left(a^{r-1}\right) x\right)=\varphi\left(\varphi\left(a^{r-1}\right)\right) \varphi(x)=\varphi\left(a^{r-1}\right) \varphi(x) .
$$

Consequently, $\varphi\left(a^{r-1}\right) x=a^{r-1} x$ for any $x \in C_{r, m}$. Therefore,

$$
\begin{gathered}
\mathcal{A} \circ \mathcal{M}=\left\langle\left\{\varphi\left(a^{r-1}\right)\right\}\right\rangle \circ \mathcal{M}=\mathcal{M} \circ\left\langle\left\{\varphi\left(a^{r-1}\right)\right\}\right\rangle=\mathcal{M} \circ \mathcal{A}, \\
\mathcal{B} \circ \mathcal{M}=\left\langle C_{m}\right\rangle \circ \mathcal{M}=\mathcal{M} \circ\left\langle C_{m}\right\rangle=\mathcal{M} \circ \mathcal{B}, \\
\mathcal{C} \circ \mathcal{M}=\left\langle C_{m}\right\rangle \circ \mathcal{M}=\mathcal{M} \circ\left\langle C_{m}\right\rangle=\mathcal{M} \circ \mathcal{C},
\end{gathered}
$$

for any $\mathcal{M} \in N_{<\omega}\left(C_{r, m}\right)$ and thus $\mathcal{A}, \mathcal{B}, \mathcal{C} \in C\left(N_{<\omega}\left(C_{r, m}\right)\right)$.

Consider the finite monogenic semigroup $C_{2, m}=\left\{a, a^{2}, \ldots, a^{m+1} \mid\right.$ $\left.a^{m+2}=a^{2}\right\}$. In this case, $x y \in C_{m}$ for any $x, y \in C_{2, m}$ and thus $\mathcal{A} \circ \mathcal{B}=N_{<\omega} \varphi(\mathcal{A}) \circ N_{<\omega} \varphi(\mathcal{B})$ for any $\mathcal{A}, \mathcal{B} \in N_{<\omega}\left(C_{2, m}\right)$. It is easy to see that $e=a^{m}$ is the identity element of the maximal subgroup $C_{m}=C_{2, m} \backslash\{a\}$. Since $\varphi(a)=e a=a^{m} a=a^{m+1}$, then $a^{m+1}$ is the unique element whose preimage under retraction $\varphi: C_{2, m} \rightarrow C_{m}$ is not a singleton. Therefore,

$$
C\left(N_{<\omega}\left(C_{2, m}\right)=\left\{\left\langle\left\{a, a^{m+1}\right\}\right\rangle,\left\langle C_{m}\right\rangle,\left\langle C_{2, m}\right\rangle,\left\langle C_{2, m} \backslash\left\{a^{m+1}\right\}\right\rangle,\langle\{g\}\rangle: g \in C_{2, m}\right\}\right\} .
$$


Problem 3.4. Given a monogenic semigroup $C_{r, m}, r>2$, describe the center of the semigroup $N_{<\omega}\left(C_{r, m}\right)$.

\section{Right and Left Cancelable Elements}

In this section, we shall detect right and left cancelable elements of extensions $N_{<\omega}\left(C_{r, m}\right)$ of finite monogenic semigroups $C_{r, m}$.

Proposition 4.1. The extension $N_{<\omega}\left(C_{r, m}\right)$ has (left, right) cancelable elements if and only if the index $r$ of a monogenic semigroup $C_{r, m}$ is equal to 1.

Proof. Let $r>1$ and $a$ be the generator of a semigroup $C_{r, m}$. Consider the map $\varphi: C_{r, m} \rightarrow C_{m}, \varphi: x \rightarrow e x$, where $e$ is the neutral element of the cyclic group $C_{m}$. As we showed in the proof of Theorem 3.3, $\varphi\left(a^{r-1}\right) x=a^{r-1} x$ for any $x \in C_{r, m}$.

Let $\mathcal{M}$ be a centered upfamily on a semigroup $C_{r, m}$. Then we obtain $\left\langle\left\{a^{r-1}\right\}\right\rangle \circ \mathcal{M}=\left\langle\bigcup_{a \in\left\{a^{r-1}\right\}} a * M_{a}:\left\{M_{a}\right\}_{a \in\left\{a^{r-1}\right\}} \subset \mathcal{M}\right\rangle=\left\langle a^{r-1} M: M \in \mathcal{M}\right\rangle$ $\left.=\left\langle\varphi\left(a^{r-1}\right) M: M \in \mathcal{M}\right\rangle=\left\{\varphi\left(a^{r-1}\right)\right\}\right\rangle \circ \mathcal{M} \quad$ and $\quad \mathcal{M} \circ\left\langle\left\{a^{r-1}\right\}\right\rangle=$ $\left\langle\bigcup_{a \in M} a *\left\{a^{r-1}\right\}: M \in \mathcal{M}\right\rangle=\left\langle M a^{r-1}: M \in \mathcal{M}\right\rangle=\left\langle M \varphi\left(a^{r-1}\right): M \in \mathcal{M}\right\rangle$ $=\mathcal{M} \circ\left\langle\left\{\varphi\left(a^{r-1}\right)\right\}\right\rangle$. Since $a^{r-1} \neq \varphi\left(a^{r-1}\right)$, the centered upfamily $\mathcal{M}$ is neither left nor right cancelable.

If $r=1$, then a monogenic semigroup $C_{1, m}=C_{m}$ is a group. Let $e$ be the neutral element of the group $C_{m}$. Then $\langle\{e\}\rangle \circ \mathcal{M}=\mathcal{M}=\mathcal{M} \circ\langle\{e\}\rangle$ for any $\mathcal{M} \in N_{<\omega}\left(C_{m}\right)$, and equalities $\chi \circ\langle\{e\}\rangle=\mathcal{Y} \circ\langle\{e\}\rangle,\langle\{e\}\rangle \circ \chi=\langle\{e\}\rangle \circ \mathcal{Y}$ imply that $\chi=\mathcal{Y}$. Consequently, the principal ultrafilter $\langle\{e\}\rangle$ is a cancelable element of the semigroup $N_{<\omega}\left(C_{1, m}\right)$. 
If $G$ is a group, then the formula

$$
\mathcal{L} \circ \mathcal{M}=\left\langle\bigcup_{a \in L} a * M_{a}: L \in \mathcal{L},\left\{M_{a}\right\}_{a \in L} \subset \mathcal{M}\right\rangle
$$

implies that the product $\mathcal{L} \circ \mathcal{M}$ of any two centered upfamilies $\mathcal{L}$ and $\mathcal{M}$ is a principal ultrafilter if and only if both $\mathcal{L}$ and $\mathcal{M}$ are principal ultrafilters. Therefore, we deduce the following proposition:

Proposition 4.2. For a group $G$, the set $N_{<\omega}(G) \backslash\{\langle\{g\}\rangle: g \in G\}$ is an ideal in $N_{<\omega}(G)$.

Lemma 4.3. A semigroup $S$ is a left (right) cancellative semigroup if and only if all principal ultrafilters are left (right) cancelable elements in the extension $N_{<\omega}(S)$.

Proof. If an element $a \in S$ is not left (right) cancelable in the semigroup $S$, then it is clear that the principal ultrafilter generated by the element $a$ is not left (right) cancelable in $N_{<\omega}(S)$.

Let $S$ be a left (right) cancellative semigroup, $a \in S$ and $\chi, \mathcal{Y} \in N_{<\omega}(S), \chi \neq \mathcal{Y}$, then without loss of generality we can assume that $X \in \chi \backslash \mathcal{Y}$ for some $X \in \chi$. Therefore, $(S \backslash X) \cap Y \neq \emptyset$ for any $Y \in \mathcal{Y}$. Since each element of $S$ is left (right) cancelable, then $(S \backslash a X) \cap a Y \neq \emptyset$ $((S \backslash X a) \cap Y a \neq \emptyset)$, and thus $\langle\{a\}\rangle \circ \chi \neq\langle\{a\}\rangle \circ \mathcal{Y}(\chi \circ\langle\{a\}\rangle \neq \mathcal{Y} \circ\langle\{a\}\rangle)$. Consequently, the left $l_{\langle\{a\}\rangle}$ (right $r_{\langle\{a\}\rangle}$ ) shift is injective and the principal ultrafilter $\langle\{a\}\rangle$ is left (right) cancelable.

Proposition 4.4. An element $\mathcal{M} \in N_{<\omega}\left(C_{1, m}\right)$ is left (right) cancelable if and only if $\mathcal{M}$ is a principal ultrafilter.

Proof. Since in any group, in particular in the cyclic group $C_{1, m}$, all elements are cancelable, then all principal ultrafilters are cancelable in the extension $N_{<\omega}\left(C_{1, m}\right)$ according to Lemma 4.3. 
Assume that some centered upfamily $\mathcal{M} \in N_{<\omega}\left(C_{1, m}\right) \backslash\left\{\langle\{g\}\rangle: g \in C_{1, m}\right\}$ is left cancelable. This means that the left shift $l_{\mathcal{M}}: N_{<\omega}\left(C_{1, m}\right)$ $\rightarrow N_{<\omega}\left(C_{1, m}\right), l_{\mathcal{M}}: \mathcal{A} \mapsto \mathcal{M} \circ \mathcal{A}$, is injective. According to Proposition 4.2 , the set $N_{<\omega}\left(C_{1, m}\right) \backslash\left\{\langle\{g\}\rangle: g \in C_{1, m}\right\}$ is an ideal in $N_{<\omega}\left(C_{1, m}\right)$. Consequently, $l_{\mathcal{M}}\left(N_{<\omega}\left(C_{1, m}\right)\right)=\mathcal{M} \circ N_{<\omega}\left(C_{1, m}\right) \subset N_{<\omega}\left(C_{1, m}\right) \backslash\{\langle\{g\}\rangle:$ $\left.g \in C_{1, m}\right\}$. Since $N_{<\omega}\left(C_{1, m}\right)$ is finite, $l_{\mathcal{M}}$ cannot be injective.

For the right cancelable elements the proof is analogous.

\section{References}

[1] T. Banakh and V. Gavrylkiv, Algebra in superextension of groups, II: Cancelativity and centers, Algebra Discr. Math. 4 (2008), 1-14.

[2] T. Banakh and V. Gavrylkiv, Algebra in superextension of groups: Minimal left ideals, Mat. Stud. 31 (2009), 142-148.

[3] T. Banakh and V. Gavrylkiv, Algebra in the superextensions of twinic groups, Dissert. Math. 473 (2010), 74.

[4] T. Banakh and V. Gavrylkiv, Algebra in superextensions of semilattices, Algebra Discrete Math. 13(1) (2012), 26-42.

[5] T. Banakh and V. Gavrylkiv, Algebra in superextensions of inverse semigroups, Algebra Discrete Math. 13(2) (2012), 147-168.

[6] T. Banakh and V. Gavrylkiv, Characterizing semigroups whose superextensions are commutative, Algebra Discrete Math. 17(2) (2014), 161-192.

[7] T. Banakh and V. Gavrylkiv, On structure of the semigroups of $k$-linked upfamilies on groups (submitted).

[8] T. Banakh, V. Gavrylkiv and O. Nykyforchyn, Algebra in superextensions of groups, I: Zeros and commutativity, Algebra Discr. Math. 3 (2008), 1-29.

[9] A. H. Clifford and G. B. Preston, The Algebraic Theory of Semigroups, Vol. I, Mathematical Surveys, 7, AMS, Providence, RI, 1961.

[10] V. Gavrylkiv, The spaces of inclusion hyperspaces over noncompact spaces, Mat. Stud. 28(1) (2007), 92-110.

[11] V. Gavrylkiv, Right-topological semigroup operations on inclusion hyperspaces, Mat. Stud. 29(1) (2008), 18-34.

[12] V. Gavrylkiv, Monotone families on cyclic semigroups, PBShSS 17(1) (2012), 35-45. 
[13] V. Gavrylkiv, Superextensions of cyclic semigroups, Carpathian Mathematical Publication 5(1) (2013), 36-43.

[14] V. Gavrylkiv, Semigroups of linked upfamilies, PBShSS 29(1) (2015), 104-112.

[15] V. Gavrylkiv, Semigroups of centered upfamilies on groups, Lobachevskii J. Math. (to appear).

[16] N. Hindman and D. Strauss, Algebra in the Stone-Čech Compactification, de Gruyter, Berlin, New York, 1998.

[17] J. M. Howie, Fundamentals of Semigroup Theory, The Clarendon Press, Oxford University Press, New York, 1995.

[18] J. van Mill, Supercompactness and Wallman Spaces, Math. Centre Tracts, 85, Math. Centrum, Amsterdam, 1977.

[19] A. Teleiko and M. Zarichnyi, Categorical Topology of Compact Hausdofff Spaces, VNTL, Lviv, 1999.

[20] A. Verbeek, Superextensions of Topological Spaces, MC Tracts, 41, Amsterdam, 1972 . 